\title{
Individuelle Computer-Rentenberechnung
}

\author{
BGH, Beschluß vom 5. Februar 1987 (I ZR 100/86)
}

\section{Leitsatz}

Bietet eine illustrierte Zeitschrift im Zusammenwirken mit einem Versicherungsunternehmen interessierten Lesern individuelle Rentenberechnungen durch den Computer des Versicherungsunternehmens in der Weise an, daß Erklärungsvordrucke, die der Zeitschriftenbeitrag enthält, ausgefült an die Redaktion der $Z$ Zitschrift zu senden sind, so verstößt diese gegen Art. 1 \& 1 RBeratG und \& 1 UWG.

\section{Paragraphen}

UWG $\$ 1$

RBeratG Art. 1 \&1

\section{Stichworte}

Computerberechnung - Rentenanwartschaften, Rentenberechnung (individuelle) - Rechtsberatungsmiß brauch; Rentenberechnung (individuelle) - Wettbewerbsverstoß

\section{Aus den Gründen}

I. Der ursprüngliche, am 15. 12. 1983 verstorbene, Kläger war zur Zeit der Klageerhebung im Jahre 1982 Rentenberater.

Die Beklagte verlegt unter anderem die Zeitschrift „B...“, in deren Ausgabe Nr. ... vom ... ein Artikel veröffentlicht wurde, dessen Überschrift lautete: „Wie sieht meine Rente aus? B... und die H... bieten Ihnen die Möglichkeit, Ihre Versorgungslücken bei der Rente zu erkennen und zu schließen." In dem Artikel wurde eine kostenlose Computerberechnung der Rentenanwartschaften angeboten.

Der damalige Kläger, der darin einen Verstoß gegen Art. $1 \S 1$ RBeratG und $\S 1$ UWG sah, hatte beantragt, die Beklagte unter Androhung der gesetzlichen Ordnungsmittel $\mathrm{zu}$ verurteilen, es zu unterlassen, in der Zeitschrift „B...“ den Lesern eine (Computer-)Rentenberechnung anzubieten.

Nach Abweisung der Klage durch das Landgericht hat das Berufungsgericht die Beklagte antragsgemäß verurteilt. Mit der zugelassenen Revision verfolgte die Beklagte ihren Klageabweisungsantrag zunächst weiter. Nach Aufnahme des Rechtsstreits durch die Erbinnen des Klägers haben die Parteien den Rechtsstreit für in der Hauptsache erledigt erklärt.
II. Über die Kosten des Rechtsstreits ist gemäß $\S$ 91a ZPO unter Berücksichtigung des bisherigen Sachund Streitstandes nach billigem Ermessen zu entscheiden. Danach sind sie der Beklagten aufzuerlegen, da diese ohne Eintritt des erledigenden Ereignisses im Rechtsstreit im vollen Umfang unterlegen wäre.

Wäre der ursprüngliche Kläger nicht verstorben und damit die an seine Person gebundene Prozeßührungsbefugnis nicht entfallen, wäre die Revision gegen das Berufungsurteil im Ergebnis erfolglos geblieben.

1. Der ursprüngliche Kläger war als Mitbewerber im Sinne des $\S 13$ Abs. 1 UWG a. F. klagebefugt. Als Rentenberater, zu dessen berufsmäßigen Aufgaben die Berechnung von Renten gehörte, war er durch das Angebot der Beklagten, kostenlose Rentenberechnungen in größerem Maßstab durchzuführen, unmittelbar betroffen. Anders als im Fall des Senatsurteils vom 13. 2. 1981 - I ZR 63/79 (GRUR 1981, 529 = WRP 1981, 385 - Rechtsberatungsanschein), in dem eine Zeitschrift nur den - unrichtigen - Anschein erweckt hatte, sie erteile Rechtsrat, hat die Beklagte im vorliegenden Fall individuelle Beratungen angeboten und damit - ungeachtet ihrer andersartigen Branchenzugehörigkeit (vgl. Senatsurteil vom 12. 1. 1972 - I ZR 60/70, GRUR 1972, 553 = WRP 1972, 195 - Statt Blumen ONKO-Kaffee) - ein Wettbewerbsverhältnis zum früheren Kläger begründet.

2. In materieller Hinsicht bedarf es keiner Prüfung, ob das Berufungsgericht den Klageanspruch aus $\S 823$ Abs. 2 BGB i.V. mit Art. $1 \S 1$ RBeratG herleiten durfte. Denn jedenfalls war die bis zum Ableben des Erstklägers gemäß $\S 13$ Abs. 1 UWG zulässige Klage nach $\S 1$ UWG i.V. mit Art. $1 \S 1$ RBeratG begründet.

Die Erwägungen, mit denen das Berufungsgericht einen Verstoß gegen Art. $1 \S 1$ RBeratG bejaht hat, lassen keinen Rechtsfehler erkennen.

Das Berufungsgericht ist zutreffend davon ausgegangen, daß die in der Zeitschrift ,B. 4 angebotene Rentenberechnung als Besorgung fremder Rechtsangelegenheiten im Sinne des Art. 1 \& 1 Abs. 1 RBeratG anzusehen ist. Dies folgt zwingend daraus, daß das Gesetz selbst (in Art. 1 \& 1 Abs. 1 Satz 2 i. V. mit Nr. 1 RBeratG) die Rentenberatung, zu der die Berechnung der Renten gehört, zu den erlaubnispflichtigen Tätigkeiten zählt.

Als rechtsfehlerfrei erweist sich auch die Annahme des Berufungsgerichts, daß es sich bei dem angegriffenen Angebot in der Zeitschrift „B...“ um ein fremdes 
Berechnungsangebot auch der Beklagten - nicht nur der H... Versicherungs AG - gehandelt habe. Dies ergibt sich - worauf das Berufungsgericht zutreffend abgestellt hat - sowohl aus dem Wortlaut des Angebots, in dem es hieß: „B... und die H... bieten Ihnen die Möglichkeit ...", als auch aus dem Umstand, daß die interessierten Leser die auszufüllenden Erklärungsvordrucke der Zeitschrift entnehmen konnten und sie an die Redaktion der Zeitschrift senden sollten. Unter diesen Umständen ist es nicht entscheidend, daß die Berechnung durch den Computer der H... Versicherungs AG erfolgen sollte und die Leser hierauf auch hingewiesen wurden. Denn die Beklagte übernahm nicht nur einen Teil der Verantwortung für die Beratung, sondern leistete mit der Bekanntgabe der Aktion sowie mit Abdruck, Sammlung und Weiterleitung der Erklärungsvordrucke einen eigenen Tatbeitrag, ohne den die als gemeinsame Aktion angekündigte Rentenberechnung für interessierte Leser - jedenfalls in der angebotenen Form - überhaupt nicht hätte stattfinden können. In Anbetracht der Bedeutung und Eigenständigkeit dieses Tatbeitrags und der nach außen bekundeten Eigenverantwortlichkeit auch der Beklagten für die angekündigte Aktion brauchte das Berufungsgericht nicht zu prüfen, ob möglicherweise - wofür allerdings nichts vorgetragen war - die H... Versicherungs AG ihrerseits im Rahmen ihrer Versicherertätigkeit zu einer Rentenberechnung gemäß Art. $1 \S 5$ Nr. 1 RBeratG berechtigt gewesen sein sollte. Denn eine solche Berechtigung ließe - wie das Berufungsgericht ebenfalls zutreffend ausgeführt hat - die Unzulässigkeit einer selbständigen und eigenverantwortlichen Mitwirkung der Beklagten an einer solchen Rentenberatungsaktion unberührt.

Das Berufungsgericht hat schließlich auch zu Recht die von der Beklagten angebotene Beratung als geschäftsmäßig im Sinne des Art. 1 \& 1 Satz 1 RBeratG angesehen. Es hat dazu rechtsfehlerfrei ausgeführt, daß es sich nicht um eine in einem Gelegenheitsfall aus besonderen Gründen ausgeübte einmalige Beratungstätigkeit, sondern um eine - wenn auch zeitlich befristete - auf eine Vielzahl von Fällen angelegte Aktion gehandelt habe. Bereits die Dauer dieser erstmaligen Aktion - vom 11. 3. bis 15. 4. 1982 - lasse die Beratungstätigkeit als geschäftsmäßig erscheinen, selbst wenn die Beklagte noch nicht geplant haben sollte, die Aktion zu wiederholen.

Die Verletzung der Vorschrift des Art. $1 \S 1$ Abs. 1 RBeratG stellt zugleich einen Verstoß gegen $\S 1$ UWG dar. Ergänzender Feststellungen des Berufungsgerichts hätte es hierzu nicht bedurft. Die Sittenwidrigkeit ergibt sich nämlich daraus, daß der Erlaubniszwang für rechtsbesorgende Tätigkeiten nicht nur auf wertneutralen Vorschriften beruht, sondern im allgemeinen Interesse an einer zuverlässigen Rechtspflege liegt (BGHZ 48, 12, 17; BGH Urt. v. 18. 1. 1974 - I ZR 13/73, GRUR 1974, 396, 398 = WRP 1974, 204 - Unfallhelferring II; st. Rspr.).

\section{ZIVILRECHT}

\section{Koppelung von Hardware und Software}

\section{BGH, Urteil vom 25. März 1987 (VIII ZR 43/86)}

\section{Amtliche Leitsätze}

1. Zur Rückabwicklung eines Software-Überlassungsvertrages aufgrund einer positiven Vertragsverletzung des Lieferanten (Lizenzgebers) durch Einbau einer Programmsperre und deren Benutzung als Druckmittel zum Abschluß eines Wartungsvertrages.

2. Die Zusammenfassung zweier Vereinbarungen über den Kauf eines Computers (Hardware) und die zeitlich nicht begrenzte Überlassung von Software (als Lizenzvertrag) in ein und derselben Vertragsurkunde kann eine Vermutung dafür begründen, daß ein einheitlicher Vertrag mit gleichen Folgewirkungen bei Störungen in einem der Teilbereiche abgeschlossen werden sollte. Sie ist widerlegt, wenn sich der Vertrag auf den Kauf eines handelsüblichen Computers und auf die Uberlassung von Standard-Software bezieht. Ist in einem solchen Falle der Softwarevertrag wegen positiver Vertragsverletzung rückgängíg zu machen oder deswegen fristlos gekündigt, wird der Hardwarevertrag davon nicht berührt.

\section{Nichtamtlicher Leitsatz}

Der Leitsatz „Sie (Die Vermutung) ist widerlegt, wenn sich der Vertrag auf den Kauf eines handelsüblichen Computers und auf die Uberlassung von Standardsoftware bezieht" gilt unter der Voraussetzung, daß Hardware und Software nicht speziell aufeinander abgestimmt sind.

\section{Paragraphen}

BGB: $\$ 139 ; \S 325 ; \S 326 ; \S 542 ; \S 581$

\section{Stichworte}

Fehler - Programmsperre; Koppelung von Hardware und Standardprogrammen; a. o. Kündigung - wegen Zerstörung des Vertrauensverhältnisses; Programmschutz; Uberlassung von Standardprogrammen rechtliche Einordnung 